Supplement of Biogeosciences, 16, 1607-1627, 2019

https://doi.org/10.5194/bg-16-1607-2019-supplement

(C) Author(s) 2019. This work is distributed under

the Creative Commons Attribution 4.0 License.

(c) (1)

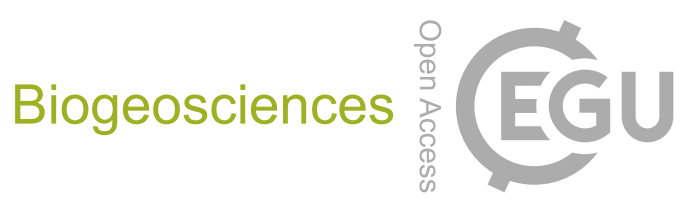

Supplement of

\title{
Cold-water corals and hydrocarbon-rich seepage in Pompeia Province (Gulf of Cádiz) - living on the edge
}

Blanca Rincón-Tomás et al.

Correspondence to: Blanca Rincón-Tomás (b.rincontomas@gmail.com)

The copyright of individual parts of the supplement might differ from the CC BY 4.0 License. 
Table S1: Relative abundance related to different bacterial taxa found in the analyzed samples.

\begin{tabular}{|c|c|c|c|c|c|c|c|c|c|c|}
\hline Taxonomy & $\begin{array}{r}\text { D10-R3 } \\
\text { _bac1 } \\
\end{array}$ & $\begin{array}{r}\text { D10-R3 } \\
\text { _bac2 } \\
\end{array}$ & $\begin{array}{r}\text { D10-R3 } \\
\text { _bac3 }\end{array}$ & $\begin{array}{r}\text { D10-R7 } \\
\text { _bac1 } \\
\end{array}$ & $\begin{array}{r}\text { D10-R7 } \\
\text { _bac2 } \\
\end{array}$ & $\begin{array}{r}\text { D10-R7 } \\
\text { _bac3 } \\
\end{array}$ & $\begin{array}{r}\text { D10-R7 } \\
\text { _bac4 } \\
\end{array}$ & $\begin{array}{r}\text { D03-B1 } \\
\text { bac1 } \\
\end{array}$ & $\begin{array}{r}\text { D03-B1 } \\
\text { _bac2 } \\
\end{array}$ & $\begin{array}{r}\text { D03-B1 } \\
\text { _bac3 } \\
\end{array}$ \\
\hline Acidobacteria & 14336 & 11021 & 15071 & 404 & 526 & 311 & 311 & 12687 & 10869 & 11616 \\
\hline Actinobacteria & 4724 & 4340 & 5322 & 562 & 709 & 729 & 757 & 3901 & 3426 & 3410 \\
\hline Bacteroidetes & 616 & 533 & 692 & 964 & 3254 & 2213 & 1946 & 114 & 200 & 153 \\
\hline Calditrichaceae & 43 & 33 & 52 & 633 & 1679 & 1334 & 2057 & 49 & 33 & 32 \\
\hline Chloroflexi & 12335 & 9326 & 15451 & 3407 & 1459 & 1424 & 2142 & 20841 & 21217 & 19102 \\
\hline Dadabacteria & 4008 & 2468 & 4064 & 8 & 3 & 5 & 0 & 2394 & 2287 & 1782 \\
\hline Sulfurovum & 6 & 6 & 2 & 406 & 465 & 268 & 256 & 0 & 0 & 0 \\
\hline Sulfurimonas & 4 & 1 & 0 & 114 & 344 & 267 & 324 & 0 & 0 & 1 \\
\hline Epsilonbacteria (others) & 0 & 1 & 1 & 25 & 58 & 37 & 56 & 0 & 0 & 0 \\
\hline Gemmatimonadetes & 1549 & 1113 & 1780 & 7 & 0 & 3 & 2 & 1256 & 1039 & 1042 \\
\hline Nitrospinae & 151 & 127 & 202 & 1 & 0 & 0 & 0 & 217 & 175 & 186 \\
\hline Nitrospira & 2920 & 1912 & 3287 & 1 & 7 & 8 & 6 & 466 & 365 & 374 \\
\hline Kaiserbacteria & 847 & 465 & 1068 & 2 & 2 & 26 & 9 & 118 & 74 & 122 \\
\hline Planctomycetes & 297 & 242 & 390 & 1132 & 1585 & 1540 & 1344 & 159 & 170 & 129 \\
\hline Poribacteria & 464 & 332 & 637 & 31 & 8 & 6 & 12 & 592 & 483 & 510 \\
\hline
\end{tabular}


Table S1. Continued

\begin{tabular}{|c|c|c|c|c|c|c|c|c|c|c|}
\hline Taxonomy & $\begin{array}{r}\text { D10-R3 } \\
\text { bac1 }\end{array}$ & $\begin{array}{r}\text { D10-R3 } \\
\text { bac2 }\end{array}$ & $\begin{array}{r}\text { D10-R3 } \\
\text { bac3 }\end{array}$ & $\begin{array}{r}\text { D10-R7 } \\
\text { bac1 }\end{array}$ & $\begin{array}{r}\text { D10-R7 } \\
\text { _bac2 }\end{array}$ & $\begin{array}{r}\text { D10-R7 } \\
\text { _bac3 }\end{array}$ & $\begin{array}{r}\text { D10-R7 } \\
\text { _bac4 }\end{array}$ & $\begin{array}{r}\text { D03-B1 } \\
\text { bac1 }\end{array}$ & $\begin{array}{r}\text { D03-B1 } \\
\text { bac2 }\end{array}$ & $\begin{array}{r}\text { D03-B1 } \\
\text { _bac3 }\end{array}$ \\
\hline Alphaproteobacteria & 2194 & 1609 & 2999 & 54 & 317 & 382 & 165 & 2593 & 2244 & 2315 \\
\hline Desulfatiglans & 2 & 0 & 0 & 895 & 1047 & 558 & 782 & 0 & 0 & 0 \\
\hline Desulfococcus & 0 & 0 & 0 & 1984 & 1878 & 590 & 1380 & 0 & 0 & 0 \\
\hline SEEP-SRB1 & 5 & 1 & 3 & 2238 & 1536 & 1352 & 1085 & 0 & 0 & 0 \\
\hline Desulfobulbus & 3 & 0 & 0 & 355 & 375 & 367 & 685 & 0 & 0 & 0 \\
\hline SEEP-SRB2 & 0 & 0 & 0 & 1199 & 1223 & 1162 & 1255 & 0 & 0 & 0 \\
\hline Myxococcales & 498 & 381 & 750 & 74 & 73 & 77 & 105 & 1509 & 1129 & 1428 \\
\hline $\begin{array}{l}\text { Deltaproteobacteria } \\
\text { (others) }\end{array}$ & 259 & 168 & 312 & 3204 & 2854 & 2174 & 2360 & 266 & 245 & 256 \\
\hline Sedimenticolaceae & 0 & 1 & 0 & 136 & 216 & 357 & 324 & 0 & 0 & 0 \\
\hline Thiohalophilus & 0 & 0 & 1 & 93 & 172 & 99 & 113 & 0 & 0 & 0 \\
\hline Enterobacteria & 28 & 13 & 44 & 1 & 0 & 0 & 0 & 847 & 1829 & 1357 \\
\hline MMG-2 & 1 & 4 & 4 & 66 & 462 & 679 & 422 & 0 & 0 & 0 \\
\hline Nitrosococcaceae & 397 & 271 & 597 & 0 & 0 & 1 & 1 & 2442 & 1907 & 2043 \\
\hline Woeseiaceae & 395 & 307 & 556 & 14 & 52 & 118 & 50 & 2025 & 2086 & 1856 \\
\hline $\begin{array}{l}\text { Thiomicrospiraceae } \\
\text { (endosymbionts) }\end{array}$ & 0 & 1 & 0 & 392 & 639 & 534 & 712 & 0 & 0 & 0 \\
\hline
\end{tabular}


Table S1: Continued

\begin{tabular}{|c|c|c|c|c|c|c|c|c|c|c|}
\hline Taxonomy & $\begin{array}{r}\text { D10-R3 } \\
\text { bac1 } \\
\end{array}$ & $\begin{array}{r}\text { D10-R3 } \\
\text { _bac2 } \\
\end{array}$ & $\begin{array}{r}\text { D10-R3 } \\
\text { _bac3 } \\
\end{array}$ & $\begin{array}{r}\text { D10-R7 } \\
\text { bac1 } \\
\end{array}$ & $\begin{array}{r}\text { D10-R7 } \\
\text { _bac2 } \\
\end{array}$ & $\begin{array}{r}\text { D10-R7 } \\
\text { _bac3 } \\
\end{array}$ & $\begin{array}{r}\text { D10-R7 } \\
\text { bac4 } \\
\end{array}$ & $\begin{array}{r}\text { D03-B1 } \\
\text { bac1 } \\
\end{array}$ & $\begin{array}{r}\text { D03-B1 } \\
\text { bac2 } \\
\end{array}$ & $\begin{array}{r}\text { D03-B1 } \\
\text { bac3 } \\
\end{array}$ \\
\hline Thiotrichaceae & 0 & 0 & 2 & 185 & 498 & 671 & 506 & 0 & 0 & 0 \\
\hline $\begin{array}{l}\text { Gammaproteobacteria } \\
\text { (others) }\end{array}$ & 4127 & 2572 & 4572 & 138 & 850 & 908 & 571 & 2103 & 1992 & 1980 \\
\hline Spirochaeta & 853 & 541 & 1021 & 344 & 442 & 410 & 256 & 293 & 254 & 301 \\
\hline Verrucomicrobia & 392 & 254 & 505 & 0 & 1 & 0 & 0 & 1037 & 1207 & 1080 \\
\hline Unclassified & 392 & 304 & 612 & 1857 & 2548 & 1680 & 1911 & 253 & 267 & 295 \\
\hline
\end{tabular}


Table S2. Relative abundance related to different archaeal taxa found in the analyzed samples.

\begin{tabular}{|c|c|c|c|c|c|c|c|c|c|c|}
\hline Taxonomy & $\begin{array}{r}\text { D10-R3 } \\
\text { _arch1 } \\
\end{array}$ & $\begin{array}{r}\text { D10-R3 } \\
\text { arch2 } \\
\end{array}$ & $\begin{array}{r}\text { D10-R3 } \\
\text { arch3 } \\
\end{array}$ & $\begin{array}{r}\text { D10-R7 } \\
\text { _arch1 } \\
\end{array}$ & $\begin{array}{r}\text { D10-R7 } \\
\text { _arch2 }\end{array}$ & $\begin{array}{r}\text { D10-R7 } \\
\text { _arch3 } \\
\end{array}$ & $\begin{array}{r}\text { D10-R7 } \\
\text { _arch4 } \\
\end{array}$ & $\begin{array}{r}\text { D03-B1 } \\
\text { _arch1 } \\
\end{array}$ & $\begin{array}{r}\text { D03-B1 } \\
\text { arch2 } \\
\end{array}$ & $\begin{array}{r}\text { D03-B1 } \\
\text { _arch3 } \\
\end{array}$ \\
\hline ANME-1 & 2 & 8 & 1 & 18976 & 3699 & 11170 & 15108 & 0 & 0 & 0 \\
\hline ANME-2 & 304 & 254 & 119 & 17049 & 9842 & 14581 & 14865 & 1 & 1 & 0 \\
\hline Thermoplasmata & 0 & 1 & 1 & 219 & 83 & 380 & 137 & 3 & 1 & 0 \\
\hline Candidatus Nitrosopumilus & 5841 & 6724 & 6074 & 3 & 1 & 1 & 1 & 2286 & 3931 & 3726 \\
\hline Cenarchaeum & 28772 & 30241 & 28018 & 0 & 0 & 0 & 0 & 30843 & 38709 & 38587 \\
\hline Nitrosophaera (others) & 2197 & 2625 & 2728 & 7 & 0 & 2 & 0 & 106 & 165 & 149 \\
\hline Asgardaeota & 0 & 1 & 0 & 475 & 156 & 493 & 450 & 2 & 0 & 0 \\
\hline Bathyarchaeia & 0 & 0 & 0 & 25 & 24 & 104 & 52 & 0 & 1 & 0 \\
\hline Woesearchaeia & 1475 & 3941 & 1015 & 35 & 8 & 20 & 30 & 13 & 17 & 10 \\
\hline Unclassified & 1477 & 3943 & 1017 & 823 & 282 & 1050 & 788 & 15 & 18 & 10 \\
\hline
\end{tabular}

